\title{
Criminologie
}

\section{Nouvelle donne législative et causes de la criminalité " corporative "}

\section{Laureen Snider}

Volume 30, numéro 1, printemps 1997

Criminalités économiques

URI : https://id.erudit.org/iderudit/017395ar

DOI : https://doi.org/10.7202/017395ar

Aller au sommaire du numéro

\section{Éditeur(s)}

Les Presses de l'Université de Montréal

ISSN

0316-0041 (imprimé)

1492-1367 (numérique)

Découvrir la revue

Citer cet article

Snider, L. (1997). Nouvelle donne législative et causes de la criminalité " corporative ». Criminologie, 30(1), 9-34. https://doi.org/10.7202/017395ar
Résumé de l'article

This paper examines the ideological and political collapse of laws regulating corporate crime in North America. In an era where social control and criminalization are steadily increasing, corporate crime has been normalized, shorn of its negative, criminal implications, de-regulated in law. The paper asks why this has happened, looking first at the century-long battle waged by labour and other counter-hegemonic groups to censure and control the antisocial acts of corporations through the passage of criminal legislation. Second, it examines the role criminology as a discipline played in this process, and the subsequent replacement of criminological discourse and influence by the newly-ascendent law and economics movement, which has provided the much of the academic support for de-regulation. Both developments, it is argued, are linked to changes in global capitalism and the weakened nation-state. Finally, the paper argues that the removal of regulation through criminal or administrative law, and of its accompanying rhetorics of denunciation, has grave consequences for social policy. The structural and ideological forces of global capitalism that have normalized corporate crime have also provided ideal conditions for increases in its incidence and impact.
Ce document est protégé par la loi sur le droit d'auteur. L'utilisation des services d’Érudit (y compris la reproduction) est assujettie à sa politique d'utilisation que vous pouvez consulter en ligne.

https://apropos.erudit.org/fr/usagers/politique-dutilisation/ 


\section{NOUVELLE DONNE LÉGISLATIVE ET CAUSES DE LA CRIMINALITÉ « CORPORATIVE »}

Laureen Snider'

This paper examines the ideological and political collapse of laws regulating corporate crime in North America. In an era where social control and criminalization are steadily increasing, corporate crime has been normalized, shorn of its negative, criminal implications, de-regulated in law. The paper asks why this has happened, looking first at the century-long battle waged by labour and other counterhegemonic groups to censure and control the antisocial acts of corporations through the passage of criminal legislation. Second, it examines the role criminology as a discipline played in this process, and the subsequent replacement of criminological discourse and influence by the newly-ascendent law and economics movement, which has provided the much of the academic support for de-regulation. Both developments, it is argued, are linked to changes in global capitalism and the weakened nation-state. Finally, the paper argues that the removal of regulation through criminal or administrative law, and of its accompanying rhetorics of denunciation, has grave consequences for social policy. The structural and ideological forces of global capitalism that have normalized corporate crime have also provided ideal conditions for increases in its incidence and impact.

Il est ironique d'écrire un article sur le crime « corporatif » à une époque où son existence même est remise en question. Les États qui jadis édictaient des lois et mettaient sur pied des agences pour dépister, surveiller et régler les affaires illégales abrogent aujourd'hui leurs lois, sapent les agences responsables de la prévention, de la découverte et de la punition du crime «corporatif » et redonnent aux contrevenants le droit d'enfreindre la loi, gratuitement ou au bénéfice d'autorisations dûment obtenues. Ce qui, en matière de règlement, passait pour du laxisme dans les années 1970 et au début des années 1980, apparaît maintenant comme du zèle. À cette époque,

1. L'auteur est professeur au Department of Sociology, Queen's University, Kingston. Ontario, K7L 3N6. 
au moins, une réglementation contre les compagnies trop âpres au gain était instaurée par les gouvernements (et parfois même appliquée). À l'inverse, aujourd'hui nous assistons à la destruction systématique de la réglementation à travers le monde occidental tandis que la religion du marché et le langage de la gestion des risques remplacent les anciens concepts de crime et de punition.

Pourquoi cela s'est-il produit et pourquoi est-ce si important ? L'existence du crime « corporatif » tient à la définition que l'on en donne. Cette définition recouvre un ensemble de comportements choisis parmi un vaste éventail d'actes commis par des humains impliqués dans des entreprises économiques et par les organismes qui les emploient. Pour être proscrits par la loi, de tels actes doivent être définis comme dommageables et les corps législatifs doivent être convaincus qu'il faille prendre des mesures contre ces actes. Des dizaines d'études historiques ont démontré que la criminalisation de fautes traditionnelles telles que le vagabondage, le vol et les voies de fait (Chambliss, 1964 ; Hay et coll., 1975 ; Thompson, 1975) ou, plus récemment, le viol, le harcèlement sexuel et la conduite en état d'ébriété (Clark et Lewis, 1977 ; Dobash et Dobash, 1975 ; Brownmiller, 1975 ; Dutton, 1984 ; Egan, Gardner et Persad, 1988) n'a été établie que lorsque des groupes de gens déterminés, organisés et influents eurent réussi à convaincre le gouvernement que de telles lois se révélaient nécessaires.

Il fut beaucoup plus difficile, par ailleurs, de convaincre les corps législatifs de proscrire et de sanctionner les actes commis par de puissants individus ou organismes en quête de profits (Carson, 1980a, 1980b ; Bliss, 1974). L'interdépendance complexe de l'État et du capital au sein des systèmes démocratiques et la nécessité de préserver entre eux une bonne entente tout en renforçant les rapports de production générateurs de profits rendent les États réticents à l'idée de réglementer le gain en capital (Gough, 1979). La criminalisation des actions profitables des compagnies remettait en question non seulement la bonne volonté et les contributions financières des gens puissants, mais aussi les privilèges et les présupposés de la maximisation des profits. Les défenseurs du monde des affaires profitaient de la dépendance des politiciens, menaçant de fermer boutique ou de relocaliser leur compagnie lorsque l'adoption de moyens de contrôles étaient envisagée. C'est à ce fameux danger de tuer la poule aux oufs d'or que l'on distingue les lois incriminant le crime «corporatif » des législations traditionnelles. Et c'est cette différence qui fait tout particulièrement problème. Les multinationales ne s'écrouleront pas et l'ordre hégémonique ne s'effondrera pas si les cadres sont accusés de violence conjugale ou d'abus de drogues; les fondements de telles accusations criminelles sont individuels. Par contre, si les compagnies et leurs cadres sont accusés de crimes « corporatifs » (salaires en deçà du salaire minimum, conditions de travail dangereuses, falsification 
de données sur la sécurité des produits ou négligence dans le processus d'élimination des déchets dangereux), les conséquences (perte d'emplois, baisse de rentabilité, relocalisations et amoindrissement de la masse taxable) se feront alors sentir.

Ce sont les conséquences économiques, politiques et peut-être même idéologiques, et non le pouvoir personnel et l'influence politique des dirigeants d'entreprises (aussi formidables qu'ils fussent), qui expliquent pourquoi la lutte pour la criminalisation des crimes « corporatifs » a duré si longtemps. C'est la principale raison pour laquelle les discours légitimant la maximisation des profits sont plus populaires que les autres. La lutte a été longue et difficile dans chaque État, les forces favorables à la réglementation connaissant plus de défaites que de victoires. Il fut nécessaire de prouver d'abord que des actes « corporatifs » pouvaient être dommageables, ensuite que l'on pouvait y remédier, qu'il ne s'agissait pas de conséquences inévitables du processus industriel, que les employeurs (et non les employés) en étaient responsables et, finalement, que l'intervention de l'État, par de nouvelles lois, était le seul ou le meilleur moyen. Chaque étape de ce processus fut violemment contestée, et la législation ne fut habituellement adoptée qu'au terme de crises majeures impliquant plusieurs victimes. Par exemple, deux cents personnes ont dû s'empoisonner avant que le RoyaumeUni n'adopte le Food and Drink Act en 1860 (Paulus, 1974). De nos jours, la quantité de preuves requises pour remettre en question les procédés industriels nécessite des Commissions royales d'enquête, des douzaines d'études scientifiques, des groupes de pression et un important appui médiatique ; tout cela pour obtenir des lois rarement appliquées, comme celle qui régit le volume des substances cancérigènes pouvant être déversées dans les égouts, ou pour établir le fait que mettre la vie de ses employés en danger dans le but de faire un profit est en soi un acte criminel !

La criminalisation de pratiques du milieu des affaires remettait en question «l'œuf d'or » pondu par le capitalisme et renversait ainsi les hiérarchies de croyances communément partagées comme, en l'occurrence, celle de la crédibilité sous-jacente au sens commun. Encore aujourd'hui, seuls certains types de personnes sont perçus comme étant les causes des dégâts sociaux : le pauvre et le jeune, l'homme de race noire et l'autochtone sont les archétypes du criminel de même que le vol et les voies de fait représentent la quintessence du crime. Les cadres supérieurs, éminemment respectables, et les compagnies multi-milliardaires ne correspondent pas à ces stéréotypes. Il fallait des preuves accablantes pour aller à l'encontre des préjugés et s'en prendre aux bases des structures du pouvoir.

Néanmoins, au cours du dernier siècle, on a assisté à un progrès idéologique considérable, particulièrement dans les démocraties industrialisées 
occidentales ${ }^{2}$. L'idée selon laquelle un procédé doit blesser ou polluer pour être profitable - et son corollaire, à savoir que tout acte profitable est une pratique acceptable - (voir Bliss, 1974) ne fait plus l'unanimité chez les décideurs et le grand public. Des réseaux régulateurs qui contrôlent et sanctionnent l'industrie ont été établis, les produits manufacturiers sont devenus plus sécuritaires, la qualité de l'air et de l'eau s'est améliorée, le nombre d'accidents de travail et de décès a baissé (Tucker, 1990 ; Yeager, 1991). Le salaire moyen de la classe ouvrière a augmenté. Ces réformes, de concert avec des normes de santé publique et d'hygiène, ont contribué à l'augmentation de l'espérance de vie, à la bajsse de la mortalité infantile et à l'amélioration générale de la santé (Paulus, 1974 ; Carson, 1970 ; Tucker, 1990 ; Gunningham, 1984).

Ce progrès est toutefois menacé par la redéfinition de la responsabilité et de la culpabilité « corporative » qui s'amorce. Des concepts tels que celui de la "gestion des risques" redéfinissent le crime "corporatif» idéologiquement, politiquement et structurellement, en présentant des actes d'acquisition dangereux comme de simples conséquences inéluctables. Enferrés dans des vérités absolues et drapés du prestige des sciences naturelles, les défenseurs des affaires et les fonctionnaires affirment que le capital n'est dommageable pour le client-consommateur que de façon occasionnelle et fortuite. (Le citoyen est évincé du discours officiel.) Le capital reprend le terrain perdu et les dangers de retourner " au bon vieux temps ", où les travailleurs n'avaient aucun droit moral ou légal, où les coûts environnementaux n'étaient pas complètement comptabilisés et où les hommes d'affaires (sic) ne se trompaient jamais, sont bien réels. Il n'y a aucune raison de croire que l'inclination à commettre des crimes « corporatifs » a disparu avec l'arrivée de la réglementation ; les causes en sont complexes et comportent de multiples facettes, mais les impératifs de la maximisation des profits sont toujours présents au sein des transnationales postfordiennes issues de la mondialisation du capital. À cela s'ajoute qu'il y a davantage d'occasions en raison de l'abolition des sanctions, légales ou morales. En conséquence, l'espérance de vie décroîtra et le nombre de décès augmentera suite à des blessures et des maladies qui auraient pu être prévenues ; les cas de contamination du sol, de l'air et de l'eau se multiplieront et le nombre de fraudes ira en augmentant.

Notre réflexion porte sur la normalisation des crimes « corporatifs » des quinze dernières années et elle fait le lien entre les luttes idéologiques et les changements de structure, entre ja montée du capital et le déclin de l'État. Il est donc question ici notamment du démantèlement des dispositifs

2. Jusqu'à un certain point, ce progrès s'est fait aux dépens des pays du tiers monde, qui ont servi de banques de main-d'ceuvre et de sites où les produits et les pratiques bannis dans les pays industrialisés pouvaient être utilisés. 
réglementaires de l'État fédéral canadien. Ces changements sont étudiés en relation avec les luttes pour la définition et le contrôle du crime « corporatif » dans les cercles universitaires, ainsi que dans le contexte du déclin de la criminologie et de la montée en force de l'économie et des sciences naturelles à la faveur de la notion de risque. Finalement, nous avons des raisons de penser que le crime « corporatif » continue de croître à l'intérieur d'un État diminué et déréglementé.

\section{ÉTAPE I : AUGMENTER LE NIVEAU DE RESPONSABILITÉ «CORPORATIVE »}

Le monde industrialisé est en proie à une contre-révolution, le capital et ses partisans tentant d'exercer l'hégémonie sur l'opinion et de réclamer la distribution disproportionnée des richesses d'autrefois. « Le patronat cherche à reprendre les gains acquis par la classe ouvrière. " (Glasbeek, 1995, p. 128.) Après la Deuxième Guerre mondiale, les pays industrialisés de l'Europe et de l'Amérique ont connu une période de prospérité sans précédent. À cela $s$ 'ajoutant que les travailleurs formèrent des groupes actifs, et que les anciens combattants se mobilisèrent politiquement, pour assurer le succès des partis politiques de gauche, on put assister à l'apothéose de l'État-providence. L'assurance-chômage, les allocations familiales et l'assurance-maladie universelle ont établi des standards minimaux de niveau de vie. Dans ce contexte, pour donner suite aux luttes du $\mathrm{XIX}^{\mathrm{e}}$ siècle en faveur de lieux de travail plus sécuritaires et de meilleures conditions de travail (Carson, 1980a, 1980b ; Tucker, 1990 ; Paulus, 1974 ; Ursel, 1992), un système complet de services, de mesures préventives et de réseaux régulateurs fut mis en place. Les coûts de fonctionnement gouvernementaux ont augmenté et les taux d'imposition aussi. La vie dans les sociétés industrialisées est devenue un peu plus facile, plus sûre et plus longue tant pour la classe ouvrière que pour les élites.

Tout ceci s'est produit en partie parce qu'on est devenu plus exigeant vis-à-vis des « corporations »; les exigences du statut de « bon citoyen corporatif » sont graduellement devenus plus rigoureux. Les devoirs liés à la « responsabilité sociale », le niveau minimal d'éthique et de souci des objectifs autres que le profit étaient imposés aux « corporations » pour qu'elles soient considérées (y compris par elles-mêmes) comme de «bons citoyens corporatifs $»$, ont été redéfinis. Les compagnies ne pouvaient plus asseoir leur légitimité simplement en ouvrant leurs portes et en offrant de l'emploi. Les employeurs qui engageaient des enfants à titre de main-d'cuvre bon marché, qui n'entretenaient pas leur machinerie ou qui n'aéraient pas leurs usines adéquatement étaient considérés comme immoraux. Ils étaient passibles de poursuites en justice et devaient rendre des comptes aux membres de l'opposition politique ou des médias. Qui plus est, ils étaient livrés au 
regard désapprobateur de leurs homologues ainsi que des clubs sociaux et des églises. C'est par étapes et après plusieurs échecs que la discrimination envers les femmes, la discrimination raciale, l'abandon de déchets dangereux et la distribution irresponsable de produits dangereux ont été ajoutés à la liste des comportements inacceptables. Dès 1980 , de tels actes enfreignaient les lois et les normes morales à respecter. On a, à ce sujet, la preuve que les grandes entreprises estimaient que de tels actes pouvaient nuire à leur réputation. Elles remédiaient immédiatement à la situation lorsque des délits étaient découverts (Fisse et Braithwaite, 1983). Les standards minimaux aussi bien éthiques que pratiques des pays industrialisés allèrent donc en augmentant.

Les comportements que l'on peut exiger du «bon citoyen corporatif », et ceux qu'on peut lui interdire sont importants, parce qu'ils aident à former les cultures « organisationnelles ». La culture de la « corporation », ses règles informelles, ses pratiques et ses attitudes internes ont un effet direct sur les valeurs et les normes de tous les employés. Les chances des aspirants d'être promus sont déterminées, en partie, par leur capacité à s'identifier à cette culture, par la conformité de leur comportement et de leurs attitudes face aux attentes « corporatives» (Gross, 1978 ; Kanungo, 1982 ; Yeager, 1995). $\mathrm{Vu}$ que les normes en vigueur dans les ateliers et celles qui le sont dans les bureaux administratifs ne sont pas les mêmes, une éthique commune est fixée par les membres de la direction et traduite dans la réalité au travers d'attentes ponctuelles et de pratiques internes ordinaires. Voilà le processus qui relie les forces des grands ensembles (droit et lutte sociale) à celles des petits (Giddens, 1991). Si les comportements qui sont récompensés ou sanctionnés dans les activités quotidiennes des employés le sont en conformité avec la loi et des normes éthiques élevées, alors les sanctions criminelles draconiennes et la surveillance d'agences externes (les formes de contrôle social les moins efficaces) ne sont pas nécessaires. La mise en place de ces normes au sein des organisations constitue un processus interactif et dialectique qui exige des normes externes rigides. Le droit criminel est particulièrement important, parce qu'il agit aux niveaux pratique et symbolique à la fois : les sanctions de l'État exposent à une pénalité financière et à une mauvaise publicité (Fisse et Braithwaite, 1983), elles lancent également le message que le comportement sanctionné est moralement inacceptable et que les sociétés responsables ne doivent pas se livrer à des actes de ce genre. Voilà pourquoi les lois et la lutte, pour la conscientisation qu'elles requièrent sont si importantes ; ce n'est pas que ces lois sont ou peuvent être appliquées uniformément et efficacement. De fait, les frais de mise en application sont élevés, les retards énormes, les condamnations rares et les sanctions trop clémentes, pour ne pas dire inexistantes (Snider et coll., 1993). Toutefois, les lois sont des composantes essentielles de la réglementation parce qu'elles modèlent des cultures éthiques à l'intérieur et à l'extérieur des organisations. 
Le but des lois est donc de créer, de nourrir et de renforcer les cultures organisationnelles non-criminogènes plutôt que les criminogènes (Braithwaite, 1989). Le contenu des cultures organisationnelles, la réalité vécue, les comportements réels qui suscitant de la honte et fixant les limites d'un comportement acceptable varient en fonction de caractéristiques particulières et locales. Les caractéristiques de la main-d'œuvre, des propriétaires, le procédé de fabrication, l'historique de l'institution et ses rapports avec les groupes communautaires auront également une influence. Toutefois, les relations sociales internes interagissent avec les règles, les valeurs et les normes qui prévalent à l'extérieur. Les limites établies, les normes créées conjointement par la société et la politique sont négociées individuellement dans chaque organisation, bien que ce processus se produise dans un environnement fixe, de manière non-déterministe, selon les règles dans un État précis, à un moment précis, qui fixent les responsabilités des entités « corporatives ». Si ces normes déclinent et si les comportements attendus du capital deviennent moins onéreux, alors les comportements renforcés et stigmatisés par la culture « organisationnelle » changent également. Tout ceci ne se passe pas simultanément ni dans toutes les organisations. Mais, avec le temps, les relations sociales internes changeront pour s'adapter aux réalités externes. C'est pourquoi le mouvement de déréglementation représente une défaite importante pour les groupes cherchant à améliorer le mode de vie et les perspectives des groupes les moins favorisés.

Les normes de comportement ne deviennent pas de plus en plus exigeantes non que pour l'industrie ; de tels changements font partie intégrante du processus de «civilisation » (Elias, 1982, 1978 ; Spirenberg, 1984). À partir du $\mathrm{Xv}^{\mathrm{e}}$ siècle, en Europe, les manières et la tenue s'affinent toujours davantage, une certaine sensibilité face à la violence et une aversion pour la douleur physique apparaissent de par les classes sociales et les nations. Ainsi, on assiste à une transformation des idées dominantes et des institutions. Toutefois, dans les nouvelles institutions capitalistes, l'économie de production et les théories évolutionnistes vulgarisées (Hobbes et Spencer) ont toujours réussi à ébranler les normes humanitaires et démocratiques. « La survie du plus fort » fût à la fois la devise et la logique des employeurs, qui ne voyaient pas de contradiction entre l'emploi en usine d'enfants de dix ans, cuvrant jusqu'à l'épuisement, et leur foi chrétienne. Les obligations légales, éthiques et morales des organismes d'affaires envers leurs employés, leurs clients et le public en général demeurent lâches. Ainsi, les compagnies sont largement exemptées des normes d'égalité salariale, vestimentaire et des normes de comportements du $\mathrm{Xx}^{\mathfrak{e}}$ siècle. Le respect interpersonnel est obligatoire. Lorsque l'on considère le progrès que les femmes et les minorités ont accompli, on doit aussi tenir compte des récents revers qu'ont connus les droits des travailleurs à un emploi et au salaire minimum (Austin and 
Dietrich, 1990 ; Rosenbalt, 1990). Si les attentes continuent à diminuer, des comportements de moins en moins éthiques vis-à-vis des employés, des communautés, de l'État et de l'environnement risquent de devenir les nouvelles normes pour atteindre l'estime de soi des individus, l'approbation sociale et la conformité légale.

\section{LA CONTRE-RÉVOLUTION CAPITALISTE}

Pour comprendre ce renversement, on doit étudier le parcours de la contre-révolution, à laquelle a donné lieu l'alliance de l'État et du capital ces deux dernières décennies. De 1950 à 1970 , le revenu per capita réel a grimpé au rythme de 2,25\% par année aux États-Unis (Palley, 1996). Durant cette période, lorsque de nouveaux marchés étaient crés, les anciens s'étendaient, le produit national brut et les profits étaient à la hausse, et l'expansion de l'État-providence n'était pas sérieusement remise en question. Une grande partie des élites « corporatives » et politiques acceptait la protection de la main-d'œuvre et les programmes sociaux, au pis aller comme un mal nécessaire, au mieux comme des innovations positives. Qu'on songe aux pensions de vieillesse, aux bonis pour la naissance d'enfants, à l'assurance médicale et l'assurance-chômage, qui ont été mis en place par des gouvernements conservateurs progressistes au Canada. Par contre, vers la fin des années 1970 et 1980, la croissance économique et les taux de profits étaient tous deux à la baisse. Suite à la première crise du pétrole, au début des années 1970 , les manufacturiers des pays industrialisés furent sérieusement menacés pour une première fois par leurs concurrents des pays de la côte du Pacifique. Des efforts importants ont été consentis pour rattraper le terrain perdu et une baisse de rentabilité s'est amorcée.

En Grande-Bretagne et aux États-Unis, les victoires électorales étaient assurées lorsque les politiciens jouaient la carte des questions raciales et du crime, fondant leur politique sur le ressentiment et les appréhensions du peuple. C'est en jetant le blâme sur les bureaucraties gouvernementales dites paresseuses et inefficaces et sur les syndicalistes qui soit-disant se graissaient la patte que les compagnies anglo-américaines fondaient leur succès dans certains milieux, tandis que c'est par des appels en faveur d'une grande nation-habile mélange d'intérêts « corporatifs» et de nationalisme, — qu'ils assuraient leur succès dans d'autres milieux. Le monde des affaires s'est mis à attaquer les droits des syndicats de s'organiser et de négocier collectivement, de faire la grève, de refuser d'effectuer des tâches dangereuses, de réclamer leur dû et de négocier leurs salaires (Calavita, 1983). Dans les années 1980, la privatisation était à l'ordre du jour au Royaume-Uni, tandis qu'aux États-Unis, qui comptaient moins d'industries et de services nationalisés, les baisses d'impôts « corporatifs » et le démantèlement des syndicats dominaient 
les programmes de la droite (Pearce et Snider, 1995). La première vague de rationalisations s'est abattue sur l'industrie. Les emplois du secteur secondaire et le capital se sont déplacés du nord et de l'est (où les syndicats étaient plus forts) vers le sud et l'ouest (où des lois anti-syndicales dites du « droit au travail » ainsi que des salaires minimum dérisoires ou inexistants avaient cours). Des discussions sur le " gaspillage » et «l'inefficacité » gouvernementale, décrivant toute taxation comme de l'oppression, sont apparues dans la presse ; de telles idées ont vite trouvé audience chez les intellectuels tout comme dans les médias populaires.

Au début, la réglementation gouvernementale des affaires était une cible de choix. Le monde des affaires s'est longtemps cru injustement accusé des péchés du capitalisme sans que soient adéquatement reconnues ses réalisations comme la prospérité, les biens de consommation, le niveau de vie plus élevé et de meilleurs emplois ${ }^{3}$. Les économistes de l'école de Chicago confortèrent l'hypothèse voulant que le contrôle des affaires par le gouvernement mènerait inévitablement à l'inefficacité économique et à une baisse de la prospérité. Ainsi, Friedman (1962) affirme que les compagnies n'ont aucune obligation morale ou sociale envers la société ; la seule discipline acceptable est celle qui est imposée par l'économie de marché. Les partisans de Posner $(1976,1977)$ récusent toute loi régulatrice, même celles qui sont mises en place pour faire face aux échecs du marché (bien que naguère considérées, avec raison, comme compatibles avec les intérêts du capital), ainsi que les lois assurant la redistribution des revenus et autres ressources. D'autres estiment que la réglementation de l'État impose des coûts directs au monde des affaires en raison des salaires des fonctionnaires, dits «bureaucrates », et des frais indirects qu'entraînerait la baisse de la productivité. Les marchés sont trop complexes pour être compris par de simples politiciens (mais non par de simples économistes). Les arguments les plus récents permettant de consolider les revendications de principe par des revendications pragmatiques ; ils affirment notamment que les nouvelles technologies, les marchés instables et le commerce global ou libre rendent la réglementation « ancienne » désuète (Harris et Carman, 1991 ; Sethi, 1991 ; Daintith, 1987; Komarynsky, 1996). Ainsi, si dans un mouvement de décriminalisation le Canada remplaça la loi relative aux enquêtes sur les coalitions par la loi sur la concurrence des années 1980, c'est que le monde

3. Nisbet (1977) attribue le manque de respect de la société à l'influence pernicieuse des «classes intellectuelles», plus spécifiquement à leur mépris pour le commerce qu'il estime enraciné dans l'incapacité des compagnies à aspirer à un résultat plus noble que le profit. La maximisation des profits n'est pas suffisamment idéaliste pour des professeurs ! Toutefois, son article, paru dans le Wall Street Journal en 1977, illustre l'ampleur du mouvement de droite. L'opinion des professeurs de la gauche ne préoccupe pas le monde des affaires aujourd'hui, ce qui donne à cet article un ton particulièrement désuet. 
des affaires parvint à convaincre le Parlement que l'ALENA (Accord de libreéchange nord-américain) empêcherait à l'avenir la constitution de monopoles et la restriction des échanges grâce à une concurrence toujours plus grande (Snider, 1993, pp. 109-110 ; Canada, 1989).

Les attaques contre la réglementation se poursuivent, cette demière étant dénoncée pour son «immoralité » et, bien que ces coûts soient impossibles à chiffrer (comme Posner l'admet, 1976, 1977), on soutient que les coûts générés par l'application de la réglementation fédérale des États-Unis représentant $47 \%$ du budget fédéral, alors qu'ils atteignaient de $40 \%$ en 1988 (éditorial dans The Economist, repris dans le Globe and Mail, Jundi 29 juillet, 1996, p. A15) ${ }^{4}$. La réglementation est un affront, une menace pour la liberté, une taxe cachée que les politiciens sont trop lâches pour imposer ouvertement. "Ils [les politiciens] ont deux façons de dépenser votre argent : ils peuvent vous le prendre et le dépenser directement; ou ils peuvent réglementer. Un gouvernement qui réglemente refile l'addition aux consommateurs, aux employés et aux propriétaires " (The Economist, répris dans le Globe and Mail, lundi 29 juillet, 1996, p. A15).

Ce courant idéologique et politique a surtout eu des effets en 1990, particulièrement aux États-Unis ${ }^{5}$. Lorsque le boom des années 1980 a fait place à la récession des années 1990 , les déficits gouvernementaux sont devenus une cible de choix de la droite. La réduction du déficit a donné lieu à un nouveau courant visant cette fois les programmes sociaux de l'Étatprovidence. Dans l'économie américaine, les taux d'imposition « corporative " à la baisse, les abris fiscaux de certains privilégiés et les dépenses militaires, à la hausse, des années 1980 ont entraîné une dette gouvernementale et des déficits croissants. Les programmes sociaux, dits trop généreux, furent pointés du doigt, surtout les prestations du bien-être social et de l'assurance-chômage. Au Canada, de meilleurs programmes sociaux, une assurance-maladie universelle, un appareil gouvernemental aux coûts d'opération et aux taxes plus élevés, l'influence américaine, la peur des fluctuations du dollar canadien, la crainte que des industries et des emplois s'en aillent en des lieux meilleur marché, ont contribué à faire de la réduction du

4. Bien que cette statistique soit douteuse, elle reflète davantage les coupures de dépenses fédérales dans les programmes sociaux aux États-Unis que l'augmentation des coûts de la réglementation. Toutefois, la source de la citation n'est pas donnée de sorte que la vérification en est impossible.

5. Les différences entre pays sont encore substantielles. Par exemple, la NouvelleZélande a commencé à adopter des mesures de prospérité de droite (privatisation, coupures dans l'assurance-maladie, l'éducation et la réglementation, obsession de réduire le déficit) 10 ans avant le Canada. L'Australie et les pays comme la France et l'Allemagne n'ont pas sabré dans les dépenses du secteur public ou du pouvoir, mais une forte pression est exercée par les forces de la droite à l'extérieur et à l'intérieur de chaque État-nation. 

«CORPORATIVE »

déficit une priorité gouvernementale. À l'aube des années 1990 , les gouvernements de gauche et de droite, provinciaux et fédéraux, ont répondu à l'appel.

De plus, la récession des années 1990, les développements de la technologie de l'information et l'avènement d'un capitalisme réellement global, ont fourni le prétexte et la raison d'un nouveau redimensionnement « corporatif ». Depuis que General Electric a atteint des profits records en mettant à pied des milliers d'employés du jour au lendemain, au milieu des années 1980. cette tactique est devenue le raccourci à la mode pour faire monter la valeur des actions, fût-ce à court terme. Les redimensionnements disciplinent la main-d'cuvre actuelle et future, ce qui est toujours utile pour le gain de capital. Avec l'avènement de l'ordinateur et des nouvelles technologies de communication, les industries se sont transformées. Les nouvelles « corporations post-modernes » postfordiennes mettent l'accent sur la décentralisation, le travail d'équipe et l'implication de la direction. Depuis que plusieurs tâches, autrefois effectuées à l'interne sont accordées en sous-traitance. on a besoin de moins de directeurs et de coordonnateurs (Pearce et Snider, 1995 ; Keane, 1995 ; Tombs, 1995). Vers le milieu des années 1990, les profits « corporatifs » sont montés en flèche, le marché boursier a connu un boom et la relance économique sans embauche a fait son entrée. Il était possible pour les organisations de se débarrasser de leurs employés grâce aux progrès technologiques ; c'était devenu nécessaire puisque, disait-on, le capitalisme global ne permettait qu'aux « plus coriaces » de survivre ; c'était aussi devenu souhaitable, parce que le capital avait enfin réussi à se libérer de toutes les obligations ne consistant pas à faire des profits. En réalité, la montée du capital fut si complète que les nations, provinces et États se firent concurrence pour pouvoir offrir les concessions les plus généreuses, tout en demandant le moins possible en retour. Nous voici revenus au XIX siècle.

La globalisation et le libre-échange ont conduit à la « vidange » de l'État" providence (Jessop, 1993, pp. 7-8). Étant donné que les gouvernements sont incapables de restreindre ou de diriger les mouvements de capitaux, ceux-ci se déplacent là où les profits sont les plus importants, canaliséss par les marchés de valeurs mobilières. Ceci limite énormément la capacité des pays à réglementer les multinationales ou à protéger les travailleurs, les citoyens ou les consommateurs par des lois nationales. On craint d'ailleurs que de telles initiatives causent l'exode des capitaux, la fuite des investissements et des emplois, l'augmentation des déficits et la diminution de la valeur de l'argent. (C'est ce qui s'est produit au Mexique à la fin de 1993, avec des effets désastreux.) Les pouvoirs de l'État-nation ont été encore plus affectés par les traités de libre-échange tels que ceux de la CEE et l'ALÉNA. Les pouvoirs qui ne sont pas subtilisés par les marchés de change leur sont cédés 
volontairement. Les tarifs douaniers, les subventions à des travailleurs particuliers ou à des régions, les lois garantissant l'assurance-maladie ou les salaires ou qui restreignent les mouvements des « corporations » nationales peuvent tous être remis en question en tant que possibles transgressions de tels accords.

\section{LES SERVICES PUBLICS DU CANADA, SANS SERVICES}

Nul besoin d'aller voir ailleurs que dans les services publics canadiens quels sont les effets de la rationalisation, de la réduction du déficit et de la contre-révolution du capital. En 1992, le gouvernement fédéral comptait 20000 fonctionnaires chargés de faire appliquer au moins 800 réglements et amendements. La structure réglementaire qu'ils administraient, un produit tributaire des compromis et des crises d'après-guerre, était loin d'être parfaite. Elle était peu cohérente, la plupart des agences étaient « captives", dans une mesure plus ou moins grande des industries qu'elles devaient contrôler et le niveau d'efficacité variait énormément (Snider, 1978). Toutefois, la structure réglementaire pouvait être remise en question par les groupes sociaux et les membres de l'opposition lorsqu'elle manquait à ses promesses. Elle sanctionnait néanmoins les contrevenants les plus fameux (fussentils parfois parmi les plus petits et les plus faibles). Et, qui plus est, les standards minimaux du «bon citoyen corporatif », et les points de repère référant aux normes dominantes des sous-cultures organisationnelles étaient reconnus dans tout le pays. Comme nous l'avons souligné, vers le milieu des années 1980, la réglementation fut attaquée de toutes parts (voir Snider, 1993, pp. 146-159). Mais c'est en 1985, que la Loi sur la protection de l'environnement fut adoptée, par un gouvernement conservateur de surcroît. Les discours véhiculés par la presse et le Parlement de l'époque voyaient dans les coupures de la réglementation une menace pour la sécurité et le bien-être des citoyens. À peine dix ans plus tôt, l'expression « réglementation efficace » avait pas l'air d'être un oxymuron.

En 1996, les propos, attitudes et politiques du gouvernement se sont transformés. Malgré les promesses électorales de 1992, une des premières initiatives du gouvernement libéral fut l'adoption d'une loi improprement nommée Regulatory Efficiency Act. Selon cette loi, tous les ministères devaient revoir chacun de leurs règlements et démontrer au Conseil du Trésor qu'ils possédaient les ressources adéquates pour les appliquer totalement. (Abstraction faite des problèmes épistémologiques que pose le concept de totale application, jamais aucune agence, pas même les corps policiers, pourtant généreusement financés, n'a eu ce pouvoir.) Le message de déréglementation fut renforcé par des coupures massives dans les budgets ministériels. 
L'attitude vis-à-vis du monde des affaires a également changé de façon dramatique. Tandis que les adjoints ministériels et les élus ont toujours traité le capital avec la déférence requise par le pouvoir, les fonctionnaires chargés de la réglementation se sont quant à eux montrés moins confiants (Shapiro, 1984 ; Braithwaite, 1984). Cependant, les discours d'aujourd'hui sont respectueux, jusqu'à la servilité. Les fonctionnaires des ministères-clés clament maintenant plutôt que de tenter de le cacher que le principal objectif de la réglementation est de promouvoir l'efficacité maximale du marché (Bell, 1996 ; Savage, 1996). Le conflit d'intérêts fondamental et indéracinable créé d'une part par l'obligation du monde des affaires de maximiser ses profits, et, d'autre part, par celle, du gouvernement, de protéger le citoyen, force les autorités à conserver leur pouvoir de faire opposition au capital, bien que ledit conflit demeure non reconnu, ignoré et nié. Même les justifications ljbérales classiques de l'action de l'État selon lesquelles les gouvernements protègent le capital de ses propres excès pour maintenir un certain consensus, sont absentes du débat.

La nouvelle théorie envahit presque tous les documents ayant été émis au cours des cinq dernières années. On la retrouve dans sa forme la plus pure dans les rapports des fonctionnaires, comme ceux du secrétaire du Conseil privé, pour qui les citoyens sont devenus des « clients » du gouvernement. Lors d'une conférence sur la réforme réglementaire commanditée par le School of Policy Studies de l'Université Queen's', en octobre 1995, l'argument le plus positif qu'un fonctionnaire du Conseil du Trésor a réussi à trouver en faveur de la réglementation, promulgée suite à de fortes pressions de militants environnementaux et de travailleurs, fut qu'elle n'était « pas nécessairement » mauvaise (Savage, 1996, p. 106). Agriculture Canada, cherchant une façon d'absorber une énorme coupure budgétaire de $25 \%$, se livre à l'évaluation de diverses réglementations, afin de savoir lesquelles doivent être éliminées ou conservées, en « consultant ses clients [du monde des affaires] ». La logique qui les guide, selon le directeur de la division des Affaires réglementaires, est celle de la stimulation de la croissance du marché, dans le but de fournir un « meilleur accès au marché » (Komarynsky, 1996, p. 29). Le ministère du Transport, responsable de la sécurité des véhicules utilisés au Canada, de même que de l'exploitation des aéroports, s'est vu demander de réduire le nombre de ses employés de 19000 à 3500 (!), et le nombre de règlements, de 700 à 350 . Les critères régissant tous ces changements font référence, selon un fonctionnaire, à quatre grands thèmes identifiés par l'administration : la globalisation, la pression de la concurrence, les nouvelles technologies requises pour demeurer compétitif et (finalement) la

6. John Ralston Saul est cité dans ce document, in les Discours Dunning, présentés à l'Université Queen's, le 9 octobre 1996. 
qualité de vie. Ici la qualité, n'est pas comprise comme le devoir du gouvernement de s'assurer que les moyens de transport soient sécuritaires, mais plutôt que « les gens voyagent plus » (Bell, 1996, p. 24).

Aujourd'hui, dans les conférences politiques, il n'est pas rare de voir des porte-parole de l'industrie et du gouvernement s'unir contre les représentants des groupes sociaux (main- d'cuvre, environnement), en appuyant mutuellement leurs positions verbalement, et signifiant par des gestes (soupirs, roulement d'yeux), à quel point ils trouvent ennuyeux de devoir écouter les arguments désuets des militants (et des intellectuels critiques... quand ils sont invités). Cette prise de position n'a rien de nouveau dans le monde des affaires : mais le gouvernement en ne faisant pas montre du besoin de se distancer des positions de l'Association canadienne des fabricants de produits chimiques ou de l'Association canadienne des manufacturiers, fournit un indice de la nouvelle prédominance du capital. Cela démontre, par contraste, l'effondrement total des positions et de l'influence anti-hégémonique et de leur influence. La légitimité, au sens où l'entend Gramsci, n'est plus contestée ; elle repose sans équivoque sur le capital et le marché.

Il ne faudrait pas se désespérer à penser que la présence de groupes d'opposition ne fait aucune différence. La signification électorale et l'attrait médiatique de divers groupes semblent perceptibles. Signalons aussi différentes positions adoptées par les ministères. Ainsi, chez Environnement Canada, le ton est beaucoup plus nuancé et défensif que dans l'Agriculture ou le Transport. On parle ici de développement durable avec pour arrière-plan, les « vingt ans de performance économique décevante des pays de l'Ouest », les "pressions sur le gouvernement ", le « scepticisme répandu quand à la capacité de gouvernements de faire les choses adéquatement et efficacement » ainsi que des problèmes politiques « moins faciles à régler » (Smith, 1996, p. 32). Une telle prudence indique que le gouvernement reconnaît que la protection de l'environnement requiert un appui considérable du grand public. Des groupes tels que le Canadian Environmental Law Association ou le Pembira Institute for Appropriate Development jouent encore un rôle important. L'accès qu'ils ont aux médias leur procure de l'influence et des droits de regard minimaux sur la paisible alliance entre l'État et le monde des affaires? ${ }^{7}$. Pour l'instant, toutefois, s'ils sont importants c'est plus pour les reculs qu'ils ont empêchés que pour les victoires remportées.

7. Récemment, les fonctionnaires fédéraux ont été gênés par la diffusion de propositions tendant à remettre la protection environnementale entre les mains des gouvernements provinciaux. Puisque ces derniers réduisent plus rapidement que leurs pairs fédéraux, que plusieurs sont pour le développement, que et tous font face à des coupures dans les paiements du gouvernement fédéral et que les revenus fiscaux sont à la baisse, les résultats de ce transfert seraient désastreux. L'Ontario, par exemple, une province naguère soucieuse de 


\section{PENDANT CE TEMPS, NON LOIN DE LÀ...}

Où étaient les intellectuels pendant que la bataille faisait rage ? Cette partie du texte est consacrée au processus par lequel les propos les plus répandus des criminologues sur le crime « corporatif », très influents dans les milieux politiques tout au long de la période d'après-guerre, sont restés sans écho au sein du mouvement dit du droit et de l'économie, et parmi les intellectuels favorables à une déréglementation. L'introduction du terme apparu tardivement, de " crime en col blanc », est généralement attribuée à Edwin Sutherland, criminologue américain respecté, qui, en 1939, consacra son discours présidentiel devant l'American Society of Criminology aux crimes commis dans le monde des affaires et la haute société. Sutherland définit le crime en col blanc comme « un délit commis par une personne de rang social élevé et respectable, dans l'exercjce de ses activités professionnelles (Sutherland, 1940,p. 1). Bien qu'il s'en prit surtout aux crimes financiers, par son intervention remarquée Sutherland rappelait aux praticiens de la criminologie, encore jeune, que les institutions respectables violaient également les lois dans leur propre intérêt. Il critiqua aussi leur obsession à ne considérer que les actes anti-sociaux des jeunes, des pauvres et des ethnies.

Bien sûr, Sutherland ne fut pas le premier à insister sur les coûts du capitalisme. Marx, Engels et plusieurs socialistes américains et britanniques du XIX ${ }^{\mathrm{e}}$ siècle, ainsi qu'un criminologue pionnier du nom de Wilhelm Bonger (1916) ont consacré leur vie à cette entreprise. À l'aube du $\mathrm{Xx}^{\mathrm{e}}$ siècle, les journalistes et les auteurs ayant pris part au mouvement du « muckraking » s'appliquaient à révéler l'avidité et la rapacité des « requins de la finance » de cette époque, par des exposés colorés qui donneront lieu à des best-sellers. Toutefois, l'impact immédiat de ces efforts sur les élites politiques et économiques de l'époque fut minime. Ainsi The Jungle (1906) d'Upton Sinclair, dépeignant l'exploitation des ouvriers des usines américaines, d'emballage de viande fut perçu comme un document comparé au fait que de la viande contaminée pouvait arriver jusque sur les tables des privilégiés et au fait que cela faisait du tort à la réputation des États-Unis dans les autres pays. La législation qui s'ensuivit visa à corriger ces problèmes.

Ces premiers efforts n'ont eu qu'un effet limité sur la criminologie populaire. Très peu de lois proscrivant ou criminalisant la pratique des affaires, c'est-à-dire les actes favorisant la maximisation des profits, étaient mises en place. Le consensus dans les élites « corporatives », politiques et culturelles voulait que de telles lois n'étaient ni requises, ni appropriées. Ainsi,

protéger l'environnement «a (récemment) adopté un des programmes les plus agressifs pour démanteler la réglementation environnementale au Canada » (Mittelstaedt, Globe and Mail. lundi 15 juillet, 1996 : A1). 
au début du siècle, les élites du monde des affaires soutenaient et, de toute évidence, croyaient que de l'équipement non sécuritaire, des lieux de travail non aérés, de la main-d'cuvre infantile, la semaine de travail de 60 heures, et les pots-de-vin versés à tout le gouvernement de l'État de la Pennsylvanie afin de favoriser les intérêts de la Standard Oil, étaient soit non dommageables, soit de la faute de quelqu'un d'autre, soit nécessaires pour demeurer compétitif (Bliss, 1974 ; Weinberg et Weinberg, 1961).

$C^{\prime}$ est ce qui rendit l'intervention de Sutherland particulièrement significative. Que des criminologues, donc des professionnels de l'étude scientifique du crime, considérant certains actes « corporatifs » comme des crimes fut un événement marquant. Les criminologues étaient des « experts 》 respectés pour leurs études des agressions, des meurtres et du viol. Le fait pour ces savants d'appliquer l'étiquette « crime » à des dommages liés à des procédés de fabrication alla à l'encontre de la notion traditionnelle de déviance. Le monde des affaires tenant une partie de sa légitimité du droit, de telles qualifications ont une portée politique et des répercussions idéologiques réelles. C'est cela, et non pas la complexité du sujet qui explique pourquoi la définition de crime en col blanc fit J'objet de débats passionnés pendant plus de 60 ans $^{8}$. Comme Nelken (1994a : 75) le dit, l'application du terme criminalité définit la dangerosité bien plus que la dangerosité ne définit la criminalité.

La définition originale de Sutherland attire l'attention sur un type d'activités qu'on ne sanctionnait habituellement pas dans les codes criminels de l'époque. À l'exception partielle des lois anti-cartels (le Sherman Act de 1890 et la loi relative aux enquêtes sur les coalitions de 1889, au Canada), la plupart des actes d'acquisition antisociaux du monde des affaires, s'ils étaient réglementés, l'étaient par des textes administratifs ou civils sans grande force. De toute évidence, la première vague de critiques (Tappan, 1947, par exemple) visa à réserver le terme à un petit nombre de comportements, qui tombaient déjà sous le coup de codes criminels de 1939, en arguant que c'était là la seule façon d'assurer « l'objectivité » scientifique. Selon les principes du positivisme, les lois criminelles sont réelles, et non pas des construits sociaux ou politiques. Sutherland, qui soutint ces principes aussi sérieusement que ses critiques, prétendait qu'il fallait aller découvrir, au-delà de la

8. Le plus proche parallèle, et c' est très intéressant, vient du mouvement féministe, où un débat fait rage concernant la définition et le domaine que recouvre le sujet des femmes battues (comment devrait-on l'appeler, en quoi est-ce que ça consiste, quel sexe est coupable ?), ainsi que le sujet du harcelement sexuel (est-ce un crime, est-ce que c'est nuisible ?). Là, la guerre politique est plutôt menée contre les forces du patriarcat que du capital, même si elles interagissent d'une façon assez intéressante. Cette thèse implique que tout groupe, du voleur à main armée au violeur, pourrait défier les idées établies sur sa criminalité s’il avait les ressources structurelles et idéologiques nécessaires. 
lettre et des lois, affirmant qu'on pouvait lire les « vraies » intentions du législateur en cherchant les comportements qui étaient officiellement proscrits et appelaient des sanctions pénales. Seules les infractions qui rencontreraient tous ces critères, seraient définies comme des crimes en col blanc.

Malgré la poursuite des offensives sur le terrain de la définition, l'apathie des intellectuels a eu plus d'influence que la critique relative à la marginalisation de l'étude du crime en col blanc au cours des décennies suivantes. L'intérêt pour ce sujet refit surface, vers la fin des années 1960 et au début des années 1970 avec l'apparition de la «criminologie critique ». Des critiques, plus ou moins rattachés à la nouvelle gauche, qui se sont attaqués à la définition de Sutherland, en arguant qu'elle était trop étroite, avancèrent l'idée que la « nuisance » était la caractéristique qui devait définir le crime. Ainsi, tout acte qui blesse, menace, prive quelqu'un de nourriture, de gîte ou de moyens de subsistance sont des crimes. Le refus d'accorder aux travailleurs des emplois sûrs et sensés ou des salaires suffisants pour satisfaire aux exigences de la santé, de même, que le refus de fournir des conditions de travail sécuritaires deviennent autant d'actes criminels équivalant légalement et idéologiquement à un vol (privation des droits à la propriété) ou à une agression (atteinte à l'intégrité corporelle) (Sheleff, 1982 ; Reiman, 1994 ; Simon et Eitzen, 1990).

La faiblesse de la gauche et de l'orientation athéorique des principaux courants criminologiques ont rendu de tels arguments quelque peu marginaux. Mais, un consensus scientifique resta difficile à trouver. Nelken (1994b : 78) identifia sept controverses liées au crime en col blanc demeurant encore non résolues : les définitions, les causes, la réglementation et le traitement, la caractérisation, l'interaction entre réglementation et causalité. l'ambivalence de la réaction sociale et la relation entre la structure et la politique. En d'autres termes, tous les éléments imaginables ! Les criminologues ne s'entendent même pas sur les modèles de classification. Ainsi, Shapiro (1984) identifia quatre types de crimes en col blanc : la fraude (utilisation de la tromperie et de la fausse représentation) ; le marché passé avec soi-même (utilisation d'un poste dans une organisation pour s'approprier ses ressources) ; la corruption (utilisation d'une position à l'intérieur de l'organisation pour diriger ses ressources vers quelqu'un d'extérieur à l'organisation); les délits réglementaires (violation de la réglementation administrative sur la conduite des affaires, sur l'utilisation de ressources publiques, ou sur les obligations du citoyen). Pour sa part, Edelhertz (1970:4-6) distingue les crimes commis par des individus en tant qu'individus (fraudes fiscales, à de l'assurance chômage ou au bien-être, par exemple), des crimes commis par des employés (fraudes contre l'entreprise) ; puis, il range les crimes « corporatifs » en deux groupes : les actes illégaux favorisant une compagnie (fausse publicité, falsification de résultats de tests de sécurité ou d'efficacité) et les crimes et les 
actes illégaux constituant l'activité principale d'une compagnie (rénovation frauduleuse, faillite frauduleuse, fausses compagnies destinées à blanchir de l'argent et fraudes sur le marché boursier).

Les définitions demeurent surtout des listes de caractéristiques descriptives, comportant habituellement les éléments suivants : (a) les crimes en col blanc sont commis dans le contexte d'une occupation légitime ; (b) la confiance de quelqu'un est trahie ; (c) la force physique n'est habituellement pas en cause (bien que des dommages physiques puissent résulter du crime) ; (d) les objectifs sont l'argent, la propriété, le pouvoir, le prestige ; (e) le but ultime de l'acte est l'obtention d'un gain. Certains considèrent que l'utilisation de son influence pour cacher le crime ou échapper à des sanctions constitue une sixième caractéristique. Essentiellement, Jes criminologues prétendent que les criminels en col blanc, contrairement aux criminels traditionnels, utilisent leur pouvoir en vue d'un gain illégal ; qu'il aille de criminels classiques ou en col blanc, leurs actes affectent toujours des victimes réelles qui souffrent de dommages réels.

Les théoriciens plus critiques continuent à distinguer crime « occupationnel » et crime « organisationnel. » Ce dernier - crime en col blanc commis par un individu ou un groupe d'individus dans le but exclusif de réaliser un gain personnel (Coleman, $1985: 8$ ) - a pour victime une organisation, publique ou privée, une compagnie ou le gouvernement. Les crimes commis par des employés tels le détournement de fonds (« corporatifs »), la fraude aux comptes de dépenses, la corruption commerciale, l'évasion fiscale et la plupart des crimes informatiques sont habituellement sanctionnés par des lois criminelles. Les crimes « organisationnels 》 (corporatifs) qui sont commis par des gens en position de confiance, profitent aussi bien à l'organisation qu'aux individus. Ces derniers crimes peuvent être divisés en cinq catégories : la fraude et la tromperie (la fausse publicité et l'évasion fiscale, par exemple); les violations des lois du marché (les tarifications excessives ou truquées) ; les délits violents (produits et procédés de fabrication nonsécuritaires) ; les pots-de-vin et la corruption (commerciale et politique) ; la violation des libertés civils (Coleman, 1989 : 13-79). La plupart de ces comportements sont illégaux, très peu sont criminels. La principale différence entre les crimes « occupationnels » et les crimes " organisationnels " réside dans le type de victimisation. Si c'est dans l'intérêt du monde des affaires de résister aux lois et aux enquêtes sur le crime " corporatif », il appuie par contre celles qui concernent des crimes « occupationnels». Il n'est donc pas étonnant que les études portant sur le crime « occupationnel » aient capté la majeure partie des fonds de recherche et aient valu du prestige à leurs auteurs au cours des dernières années, et ce surtout aux États-Unis (Hirschi et Gottfredson, 1987 ; Hollinger et Clark, 1983 ; Wheeler, Weisburd et Bode, 1982 ; Weisburd, Chayet et Waring, 1990). Il existe très peu d'études faites 
sur le crime "corporatif " $"$; elles ont été peu financées, conduites généralement selon les méthodes qualitatives plutôt que quantitatives, et principalement outre-mer.

Le soin mis à situer l'étude du crime en col blanc sur le terrain de la politique et à utiliser des définitions favorables au monde des affaires font qu'il est des plus ironiques que la criminologie ne soit que peu présente dans l'arène politique. Quoj que soient devenus, les discours criminologiques non critiques, ils n'ont jamais pu faire concurrence au droit ou à l'économie, ni à la « science", récemment fabriquée, de l'évaluation des risques. Celle-ci a évacué le concept de crime et a rendu inutiles les débats sur la faute et les dommages. Les intérêts personnels et le marché étantt les mécanismes permettant de discipliner le monde des affaires, il n'est pas nécessaire de procéder souvent à des contrôles. Les risques peuvent être évalués chimiquement ou épidémiologiquement, et quantifiés à des degrés de certitude impressionnants (Dewees et Daniels, 1986 ; Daintith, 1987). Les « lois » de l'économie garantissent que tous les joueurs se comportent de façon rationnelle, en cherchant à maximiser leurs propres intérêts ; elles laissent au gouvernement pour seul rôle celui d'un honnête courtier ou d'un médiateur. L'analyse des risques promet que l'État, le capital et les citoyens peuvent tout avoir : la prospérité en multipliant les forces de production, la sécurité par les découvertes scientifiques et technologiques, la faveur électorale et des dépenses gouvernementales peu élevées en tirant profit des intérêts du monde des affaires pour le réglementer. De telles approches correspondent parfaitement aux programmes politiques de déréglementation, au développement du droit «corporatif ${ }^{10}$ et au redimenssionnement de l'État-nation'".

9. Voir Yeager (1991) ; Calavita et Pontell (1995) ; Simpson $(1986,1996)$; Vaughan $(1982,1995)$; et Shapiro $(1984,1985$ et 1990). La seule étude empirique à grande échelle sur le crime « corporatif » aux États-Unis est celle de Clinard et Yeager, parue en 1980 sous le titre de Corporate Crime.

10. Le dernier remède pour extirper les excès de drojt corporatif est l'École contractualiste, qui perçoit la « corporation » comme un « mécanisme de réduction des coûts de transaction pour les parties contractantes " (Glasbeek, 1995 ; p. 121). Puisque ce n'est pas une creation de l'État, qu'elle n'opère pas sous ses auspices mais selon des chartes et privilèges spéciaux comme la responsabilité limitée, conférée par l'État, (le point de vue traditionnel), la réglementation gouvernementale ne serait pas légitime. La " corporation » n'est rien de plus qu'un lieu privé supplémentaire permettant aux individus de maximiser leurs propres intérêts économiques par l'entremise de contrats avec des individus ayant les mêmes inclinations.

11. Une des principales priorités du $103^{\circ}$ Congrès des États-Unis, élu en 1994, (premier Congrès à être majoritairement républicain en $\mathbf{3 0}$ ans), fut d'adopter des lojs exigeant que les ratios coôts-bénéfices soient calculés et publiés avant que toute nouvelle loi fédérale ne soit adoptée. 


\section{CONCLUSION}

Les effets de cette révolution de la droite ont été fulgurants. Tandis que les profits « corporatifs » et les marchés boursiers sont montés en flèche et que la production a augmenté (de $11 \%$ aux États-Unis), les salaires hebdomadaires ont plafonné en 1973 (données américaines) et sont en constante baisse (de 15,5 \% jusqu'ici) (Palley, 1996:45). Le chômage et l'iniquité, à l'intérieur et entre les États-nation, sont à la hausse. « La richesse totale des 358 milliardaires du monde est équivalente aux revenus réunis de $45 \%$ des citoyens (les plus pauvres) de la planète, soit 2,3 milliards de personnes". (Keegan, 1996 : D4.) De 1977 à 1991, le coefficient d'inégalité en Angleterre a augmenté de $10 \%$, et de $3 \%$ aux États-Unis. Globalement, de 1960 à 1991, les pays les plus riches ont fait passer leur part de richesse de 70 à $85 \%$, tandis que le $20 \%$ des plus pauvres a vu ses richesses passer de $2,3 \%$ à 1,4\% (Keegan, 1996). En 1991, des propriétés publiques avaient été vendues dans plus de 88 pays (Pearce et Snider, $1995: 25$ ). Au bout du compte, « les gens travaillent plus fort, font de plus longues heures, ont moins de sécurité, moins de droits et des salaires moins élevés » (Pearce et Snider, 1995 : 44.)

Le retrait de la réglementation et des discours de dénonciation qui sont associées à celle-ci a de graves conséquences sur les politiques sociales, parce que les forces idéologiques et structurelles du capitalisme global, en train de normaliser le crime " corporatif » ont créé les conditions idéales pour augmenter l'incidence et l'impact de celui-ci. La motivation n'a pas connu de déclin ; les forces culturelles, qui ont rendu le succès - défini par la richesse et le pouvoir - populaire, sont plus envahissantes que jamais. Les cadres subissent encore de fortes pressions pour réussir, donc pour augmenter leurs profits et leur pouvoir. Les directions recherchent la croissance trimestrielle plutôt que des profits « raisonnables » à terme. Quelques uns de ces actes de maximisation des profits auront des conséquences sociales dommageables ${ }^{12}$. À l'interne, avec les idéologies populaires qui légitiment des actes qui étaient autrefois illégaux, les cultures « organisationnelles corporatives » auront moins tendance à sanctionner les individus ou les groupes incriminés. De plus, ceux dont la conscience est "molle » seront moins enclins à problématiser ou rapporter de tels actes. Après tout, qui se plaignait de la discrimination, dans les usines en 1890, lorsqu'elle était considérée comme normale ? Il y des raisons de penser que les actes « corporatifs » antisociaux

12. Les études indiquent peu de différence de motivation et de personnalité entre les «contrevenants» (les accusés) et leurs collègues de travail (Coleman, 1987; Clinard et Yeager, 1980 ; Fisse et Braithwaite, 1983 ; Passas, 1990 ; Braithwaite, 1985a, 1985b, 1984 ; Shapiro, 1985, 1984 ; Varrette, 1985 ; Vaughan, 1982 ; Vandivier, 1992). Il y a donc des raisons de croire que la criminalité « corporative » est à la hausse. 
sont à la hausse. Et le charme de la déréglementation, du point de vue des normalisateurs de la droite, c'est que maintenant, personne ne regarde plus ces actes ${ }^{13}$.

Sur le plan théorique, nous estimons que le triomphe du discours des entreprises prend ses racines dans le contrôle hégémonique qu'exerce le capital sur les ressources économiques clés ainsi que sur les techniques et les rapports sociaux de la production. Les réalités structurelles forgent l'influence du discours, mais les discours forment également les réalités structurelles. Le processus est interactif et inflexible. Les gens et les institutions qui font la promotion du capital sont bien placés pour répandre des interprétations de la réalité confortant leurs intérêts. C'est ce qu'illustre la capacité des blocs de pouvoir hégémoniques - alliance des intérêts du capital et de l'État canadien - de changer d'allégeance à volonté. Lorsque les thèses sur le comportement " corporatif », définies par la discipline criminologique, ne correspondaient pas aux intérêts et aux «besoins corporatifs », les élites du capital et de l'État cessèrent tout simplement de prendre part aux débats criminologiques. L'allégeance et les fonds de recherches se sont alors déplacés. Les intellectuels n'ont pas voulu se soumettre à la nouvelle religion de l'évaluation des risques et les activistes des groupes sociaux sont devenus superflus. Les idées critiques ont cessé d'être écoutées, parce qu'elles n'avaient plus besoin d'être entendues. Le capital avait le dessus dans le supermarché des idées et la légitimité était bon marché.

Cela dit, l'histoire nous apprend que de telles victoires ne sont jamais permanentes. Même aujourd'hui l'influence anti-hégémonique de groupes d'opposition actifs dans l'arène politique (les environnementalistes, par exemple) est manifeste. Il est donc important de se rappeler l'aphorisme de Foucault qui dit que les forces sociales érigent, constituent mais aussi oppriment. Le pouvoir « corporatif » n'est pas monolithique ; les institutions et la culture, l'essence même des organisations sociales, «sont productives et répressives à la fois » (Pearce, $1989: 166)$.

\section{BIBLIOGRAPHIE}

AUSTIN R. \& S. DIETRICH, (1990), «Employer Abuse of Low-Status Workers : The Possibility of Uncommon Relief from the Common Law », in D. Kairys, ed., The Politics of Law : A Progressive Critique. New York : Pantheon : 350-59.

13. Par contre, la majorité des experts soutiennent qu'il y a un important volume de chiffres noirs non rapportés ou non découverts (Sutherland, 1940 ; Simon et Eitzen, 1990 ; Sheleff, 1982 ;Coleman, 1985 ; Clinard et Yeager, 1980 ; Edelhertz. 1970 :Carson, 1908b). Les enquêtes lancées suite à des catastrophes importantes révèlent des schèmes de délits chroniques, à long terme, remontant souvent à des dizaines d'années. 
BELL, D., (1996), "Regulators' Perspectives », in M. Mehta, ed., Regulatory Efficiency and the Role of Risk Assessment. Queen's University, School of Policy Studies, Environmental Policy Unit : 23-26.

BLISS, M., (1974), A Living Profit : Studies in the Social Organization of Canadian Business, Toronto : McClelland \& Stewart.

BLUM, R.H. (1972), Deceivers and Deceived, Springfield, Ill : C. Thomas.

BONGER, W. (1916), Criminality and Economic Conditions, Boston : Little, Brown.

BOX, S., (1983), Power, Crime and Mystification, London : Tavistock Publications Ltd.

BRAITHWAITe, J., (1989), « Criminological Theory and Organizational Crime ", Justice Quarterly, $6: 333-358 ;$ Reprinted in D. Nelken, ed., White-Collar Crime. Aldershot, U.K. : Dartmouth, 1994b : 187-212.

BRAITHWAITE, J., (1985a), «White Collar Crime " American Review of Sociology, II : 125.

BRAITHWAITE, J., (1985b), To Punish or Persuade: The Enforcement of Coal Mine Legislation, Albany : State University of New York Press.

BRAITHWAITE, J., (1984), Corporate Crime in the Pharmaceutical Industry, London : Routledge and Kegan Paul.

BRAITHWAITE, J., (1982), «Enforced Self-Regulation : A New Strategy for Corporate Crime Control ", Michigan Law Review, 80(7) : 1466-1507.

BROWNMILLER, S., (1975), Against Our Will : Men, Women and Rape, New York : Simon \& Schuster.

CALAVITA, K., (1986), « Worker Safety, Law and Social Change : The Italian Case », Law and Sociery, 20, \#20:189-229.

CALAVITA, K.. (1983), " The Demise of the Occupational Safety and Health Administration : A Case Study in Symbolic Action », Social Problems, 30, $4: 437-48$.

CALAVITA, K., and H. PONTELL, (1995), "Saving the Savings and Loans : U.S. Government Response to Financial Crime » in F. Pearce and L. Snider, eds., Corporate Crime : Contemporary Debates. Toronto : University of Toronto Press : 199-214.

Canada, Bureau of Competition Policy. (1989), Competition Policy in Canada: The First Hundred Years. Ottawa : Consumer and Corporate Affairs.

Carson, W.G., (1980a), « The Institutionalization of Ambiguity : Early British Factory Acts ", in G. Geis \& E. Stotland, eds., White Collar Theory and Research, Beverly Hills : Sage.

CARSON, W.G., (1980b), « The Other Price of Britain's Oil : Regulating Safety on Offshore Oil Installations in the British Sector of the North Sea », Contemporary Crises, $4: 239-66$.

CARSON, W.G., (1970). « White Collar Crime and the Enforcement of Factory Legislation », British Journal of Criminology, $10: 383-98$

CHAMBLISS, W., (1964), «A Sociological Analysis of the Law of Vagrancy ", Social Problems, $12: 67-77$.

CLARKE, M., (1990), Business Crime, Cambridge : Polity Press.

CLARK, L. and D. LEWIS, (1977), Rape : The Price of Coercive Sexuality, Toronto: The Women's Press.

CLINARD, M.B. and P. YEAGER, (1980), Corporate Crime, New York : Free Press.

COLEMAN, J.W., (1987), « Toward an Integrated Theory of White-Collar Crime », American Journal of Sociology, $93: 406-439$. 
COLEMAN, J.W., (1985), The Criminal Elite : The Sociology of White Collar Crime, New York : St. Martin's Press. Second Edition, 1989.

CRESSEY. D., (1953), Other People's Money: A Study in the Social Psychology of Embezzlement, Glencoe, Illinois : Free Press.

DAINTITH, T.. (1987), ed. Law as an Instrument of Economic Policy : Comparative and Critical Approaches, Berlin : Walter de Gruyter.

DEWEES, D. \& DANIELS, R., (1986), "The Cost of Protecting Occupational Health : The Asbestos Case », Journal of Human Resources, $21: 381-89$.

DOBASH R.E. and R. DOBASH, (1975), Violence Against Wives : A Case Against the Patriarchy, New York : Free Press.

DUTTON, D. (1984), The Criminal Justice System's Response to Wife Assault, Ottawa : Ministry of the Solicitor-General, Report \#26.

EDELHERTZ, H., (1970), The Nature, Impact and Prosecution of White-Collar Crime, Washington, D.C. : National Institute for Law Enforcement and Criminal Justice, Department of Justice.

EGAN, Carolyn, LINDA L. GARDNER, and Judy V. PERSAD, (1988), « The Politics of Transformation : Struggles with Race, Class and Sexuality in the March 8th Coalition », in F. Cunningham, S. Findlay, M. Kadar, A. Lennon \& F. Silva, eds., Social Movements/Social Change. Toronto : Between the Lines and the Society for Socialist Studies, pp. 20-47.

ELIAS, N. (1982), State Formation and Civilization, London : Oxford University Press.

ELIAS, N. (1978), The Civilizing Process, London : Oxford University Press.

FISSE, B. and J. BRAITHWAITE, (1983), The Impact of Publicity on Corporate Offenders, Albany, New York : State University of New York Press.

FRIEDMAN, M. (1962), Capiralism and Freedom, Chicago : University of Chicago Press.

GIDDENS, A. (1981), The Class Structure of Advanced Societies, 2nd. Edition. London : Hutchinson.

GLASBEEK, H., (1995), « Preliminary Observations on Strains of and Strains in, Corporate Law Scholarship ", in F. Pearce \& L. Snider, eds., Corporate Crime : Contemporary Debates, Toronto : University of Toronto Press : 111-131.

GOTTFREDSON, M.R. \& T. HIRSCHI. (1990), A General Theory of Crime, Stanford : Stanford University Press.

GOUGH, I. (1979), The Political Economy of the Welfare State, London: Macmillan.

GROSS, E., (1978), « Organizational Crime : A Theoretical Perspective », Studies in Symbolic Interaction, $1: 55-85$.

GUNNINGHAM, N., (1984), Safeguarding the Workers, Sydney : Law Book Co. Ltd.

HAY, D., P. LINEBAUGH, J. RULE, E. THOMPSON and C. WINSLOW. Albion's Fatal Tree : Crime and Society in 18th Century England, Harmondsworth : Penguin.

HARRIS, R. \& J. CARMAN, (1991), "The Political Economy of Regulation », in S. P. Sethi, P. Steidlmeier \& C. Falbe, eds., Scaling the Corporate Wall. Englewood Cliffs, N.J. : Prentice-Hall : 24-56.

HIRSCHI, T. \& M. GOTTFREDSON, (1987), "Causes of White-Collar Crime ", Criminology, 25 : 949-974.

HOLLINGER, R. \& J. CLARK. (1983), Theft by Employees, Toronto : Lexington Books.

JESSOP, B., (1993), « Towards a Schumpeterian Welfare State ? Preliminary Remarks on a Post-Fordist Political Economy ", Studies in Political Economy, 40 (Spring) : 7-39. 
KANUNGO, R. (1982), Work Alienation : An Integrated Approach, New York : Praeger.

KEANE, C., (1995), « Loosely Coupled Systems and Unlawful Behaviour : Organization Theory and Corporate Crime », in F. Pearce \& L. Snider, eds., Corporate Crime : Contemporary Debates, Toronto : University of Toronto Press : 168-80.

KEEGAN, V., (1996), « How Digital Billionaires Altered the Meaning of Wealth ", The Guardian. Reprinted in The Globe and Mail.. Saturday August 10 : D4.

KOMARYNSKY, M., (1996), « Regulators' Perspectives ", in M. Mehta, ed., Regulatory Efficiency and the Role of Risk Assessment, Queen's University, School of Policy Sudies, Environmental Policy Unit : 27-29.

Leigh, P., (1989), « Compensating Wages for Job-Related Death : The Opposing Argument », Journal of Economic Issues, 23, $3: 823-42$.

NELKEN, D., (1994a), ed. The Futures of Criminology, London : Sage Publications.

NELKEN, D., (1994b), ed. White-Collar Crime. Aldershot, U.K. : Dartmouth.

NISBET, R., (1977), « Capitalism and the Intellectuals », Wall Street Journal, September 16.

PALLEY, T., (1996), "The Forces Making for an Economic Collapse ", The Atlantic Monthly, Jul : 43-59.

PASSAS, N., (1990), « Anomie and Corporate Deviance », Contemporary Crises, 14 : 157 78.

PASSAS, N. \& D. Nelken, (1993), "The Thin Line between Legitimate and Criminal Enterprise : Subsidy Frauds in the European Community », Crime, Law and Social Change, $19: 223-43$.

PAULUS, I. (1974), The Search for Pure Food : A Sociology of Legislation in Britain, London : Martin Robertson.

PEARCE, F. (1989), The Radical Durkheim, London : Unwin Hyman.

PEARCE, F. \& L. SNIDER, (1995), « Regulating Capitalism », in F. Pearce \& L. Snider, eds., Corporate Crime : Contemporary Debates. Toronto : University of Toronto Press : 19-47.

POSNER, R. (1977), Economic Analysis of Law, New York : Little, Brown.

POSNER, R. (1976), Antitrust Law, Chicago : University of Chicago Press.

PRESTHUS, R. (1978), The Original Society, New York : St. Martin's Press.

REIMAN, J., (1994), The Rich Get Richer and the Poor Ger Prison, 4th edition. New York : Macmillan.

ROSENBLATT, R., (1990), "Social Duties and the Problem of Rights in the American Welfare State », in D. Kairys, ed., The Politics of Law : A Progressive Critique, New York : Pantheon : 90-114.

SANKAR, Yassin, (1994), Organizational Behavior: The Ethical Challenge, Toronto : Canadian Scholars Press.

SAVAGE, F. (1996), "Regulatory Reform Roundtable : Is Any Consensus Possible », in M. Mehta, ed., Regulatory Efficiency and the Role of Risk Assessment. Queen's University, School of Policy Studies, Environmental Policy Unit : 105-107.

SETHI, S. PRAKASH. P. STEIDLMEIER, \& C. M. FALBE, (1991), eds., Scaling the Corporate Wall : Readings in Social Issues of the Nineties, Englewood Cliffs, New Jersey : Prentice-Hall.

SETHI, S.P., (1991), « Conceptual Framework for Environmental Analyses of Social Issues and Evaluation of Business Response Patterns », in S. Prakash Sethi, P. Steidlmeier \& 
C. Falbe, Scaling the Corporate Wall: Readings in Social lssues of the Nineties, Englewood Cliffs, N.J : Prentice-Hall : 77-89.

SHAPIRO, S., (1990), « Collaring the Crime, not the Criminal : Considering the Concept of White-Collar-Crime » American Sociological Review, 55 (June) : 346-65.

SHAPIRO, S., (1985), « The Road Not Taken : The Elusive Path to Criminal Prosecution for White Collar Offenders $"$ Law and Society Review, 19(2): 179-217.

SHAPIRO, S. (1984), Wayward Capitalists. New Haven : Yale University Press.

SHELEFF, L.S., (1982), « International White Collar Crime » in Wickman, P. \& T. Dailey, (eds.) White Collar and Economic Crime, Toronto : D.C. Heath and Co : 39-58.

SIMON, D.R. and D.S. EITZEN, (1990), Elite Deviance 3rd edition. Boston : Allyn and Bacon Inc.

SIMPSON, S., (1996), “ Theoretical Developments in Corporate Crime Research ", Paper presented at American Society of Criminology Annual Meetings, Chicago, November 20-23.

SIMPSON, S., (1986), « The Decomposition of Antitrust : Testing a Multi-Level Longitudinal Model of Profit Squeeze ", American Sociological Review, 51 : 850-75.

SMITH, R., (1996), « Regulators' Perspectives », in M. Mehta, ed., Regulatorv Efficiency and the Role of Risk Assessment. Queen's University, School of Policy Studies, Environmental Policy Unit : 31-38.

SNIDER, L., (1993), Bad Business : Corporate Crime in Canada, Scarborough, Ontario : Nelson.

SNIDER. L., (1987), « Towards a Political Economy of Reform, Regulation and Corporate Crime ", Law and Policy, 9, $1: 37-68$.

SNIDER, L." (1978), "Corporate Crime in Canada : A Preliminary Report », Canadian Journal of Criminology, 20 : 142-68.

SPIRENBURG, P. (1984), The Spectacle of Suffering, Cambridge : Cambridge University Press.

SUTHERLAND, E., (1940), «White-Collar Criminality », American Sociological Review, 5 , February : 112.

TAPPAN, P., (1947), "Who is the Criminal ", American Sociological Review, $12: 96-102$.

THOMPSON, E.P., (1975), Whigs and Huniers : The Origin of the Black Act, New York : Pantheon.

THOMPSON, E.P., (1963), The Making of the English Working Class, London : Gollancz.

TOMBS, S., (1995), «Corporate Crime and New Organizational Forms ", in F. Pearce \& L. Snider, eds., Corporate Crime : Contemporary Debates, Toronto : University of Toronto Press : 132-46.

TUCKER, E., (1990), Administering Danger in the Workplace: The Law and Politics of Occupational Health \& Safery Regulation in Ontario, 1850-1914. Toronto : University of Toronto Press.

URSEL, J., (1992), Private Lives, Public Policy: 100 Years of State Intervention in the Family. Toronto : Women's Press.

URSEL, J., (1986), « The State and the Maintenance of Patriarchy : A Case Study of Family, Labour and Welfare Legislation in Canada ", in J. Dickinson and B. Russell, eds., Family, Economy and State, Toronto : Garamond, pp. 150-91.

VANDIVIER, $K_{1}$ (1992), « Why Should My Conscience Bother Me » in M.D. Ermann and R. Lundman (eds) Corporate and Governmental Deviance. Fourth Edition. New York : Oxford University Press : 205-228. 
VARRETTE, S.E., C. MEREDITH, P. ROBINSON \& D. HUFFMAN, (1985), ABT Association of Canada. White Collar Crime : Exploring the lssues. Ottawa : Ministry of Justice.

VAUGHAN, D. (1996), The Challenger Launch Decision : Risky Technology: Culture and Deviance at NASA. Chicago : University of Chicago Press.

VAUGHAN, D, (1982), «Transaction Systems and Unlawful Organizational Behaviour » Social Problems, 29(4) : 373-9.

VOGEL, D. (1986), National Styles of Regulation : Environmental Policy in Great Britain and the United States, Ithaca : Comell University Press.

VOGEL, D., (1983), «The Power of Business in America : A Re-Appraisal », British Journal of Political Science, $13: 4-41$.

WEINBERG A. \& L. WEINBERG, (1961), eds, The Muckrakers, New York : Simon \& Schuster.

WEISBURD, D., E. F. CHAYET \& E. J. WARING, (1990), "White Collar Crime and Criminal Careers : Some Preliminary Findings » Crime and Delinquency, 36(3) : 342355.

WHEELER, S., D. WEISBURD, \& N. BODE, (1982), « Sentencing the White-Collar Offender : Rhetoric and Reality ", American Sociological Review, 47 : 641-59.

YEAGER, P., (1995), « Management, Morality and Law : Organizational Forms and Ethical Deliberations $»$ in F. Pearce \& L. Snider, eds., Corporate Crime : Contemporary Debates. Toronto : University of Toronto Press : 147-67.

YEAGER, P., (1991), The Limits of Law: The Public Regulation of Private Pollution, Cambridge : Cambridge University Press. 